\title{
Correction to: CircLONP2 enhances colorectal carcinoma invasion and metastasis through modulating the maturation and exosomal dissemination of microRNA-17
}

Kai Han ${ }^{1,2+}$, Feng-Wei Wang ${ }^{1 \dagger}$, Chen-Hui Cao ${ }^{1+}$, Han Ling ${ }^{1 \dagger}$, Jie-Wei Chen ${ }^{1,3+}$, Ri-Xin Chen ${ }^{1}$, Zi-Hao Feng ${ }^{4}$, Jie Luo ${ }^{1}$, Xiao-Han Jin', Jin-Ling Duan', Shu-Man Li', Ning-Fang Ma ${ }^{5}$, Jing-Ping Yun ${ }^{1,2}$, Xin-Yuan Guan ${ }^{1,6}$, Zhi-Zhong Pan ${ }^{1,2}$, Ping Lan ${ }^{7}$, Rui-Hua $\mathrm{Xu}^{1}$ and Dan Xie $\mathrm{i}^{1,3^{*}}$

\section{Correction to: Mol Cancer (2020) 19:60 \\ https://doi.org/10.1186/s12943-020-01184-8}

Following publication of the original article [1], the authors identified some minor errors in image-typesetting in Fig. 4; specifically, the transwell invasion assay of HCT116 cells with circLONP2-overexpression shown in Fig. $4 \mathrm{~b}$.

The corrected figure is given below. The corrections do not have any effect on the final conclusions of the paper.

\section{Author details}

${ }^{1}$ Sun Yat-sen University Cancer Center; State Key Laboratory of Oncology in South China, Collaborative Innovation Center for Cancer Medicine, Guangzhou, China. ${ }^{2}$ Department of Colorectal Surgery, Sun Yat-sen University Cancer Center, Guangzhou, China. ${ }^{3}$ Department of Pathology, Sun Yat-sen University Cancer Center, Guangzhou, China. ${ }^{4}$ Department of Surgery, First Affiliated Hospital, Sun Yat-sen University, Guangzhou, China. ${ }^{5}$ Key Laboratory of Protein Modification and Degradation, School of Basic Medical Sciences, Affiliated Cancer Hospital \& Institute of Guangzhou Medical University,

The original article can be found online at https://doi.org/10.1186/s12943020-01184-8.

* Correspondence: xiedan@sysucc.org.cn

${ }^{\dagger}$ Kai Han, Feng-Wei Wang, Chen-Hui Cao, Han Ling and Jie-Wei Chen contributed equally to this work.

${ }^{1}$ Sun Yat-sen University Cancer Center; State Key Laboratory of Oncology in South China, Collaborative Innovation Center for Cancer Medicine,

Guangzhou, China

${ }^{3}$ Department of Pathology, Sun Yat-sen University Cancer Center,

Guangzhou, China

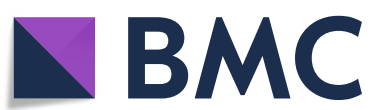

C C The Author(s). 2021 Open Access This article is licensed under a Creative Commons Attribution 4.0 International License, which permits use, sharing, adaptation, distribution and reproduction in any medium or format, as long as you give appropriate credit to the original author(s) and the source, provide a link to the Creative Commons licence, and indicate if changes were made. The images or other third party material in this article are included in the article's Creative Commons licence, unless indicated otherwise in a credit line to the material. If material is not included in the article's Creative Commons licence and your intended use is not permitted by statutory regulation or exceeds the permitted use, you will need to obtain permission directly from the copyright holder. To view a copy of this licence, visit http://creativecommons.org/licenses/by/4.0/ The Creative Commons Public Domain Dedication waiver (http://creativecommons.org/publicdomain/zero/1.0/) applies to the data made available in this article, unless otherwise stated in a credit line to the data. 
A
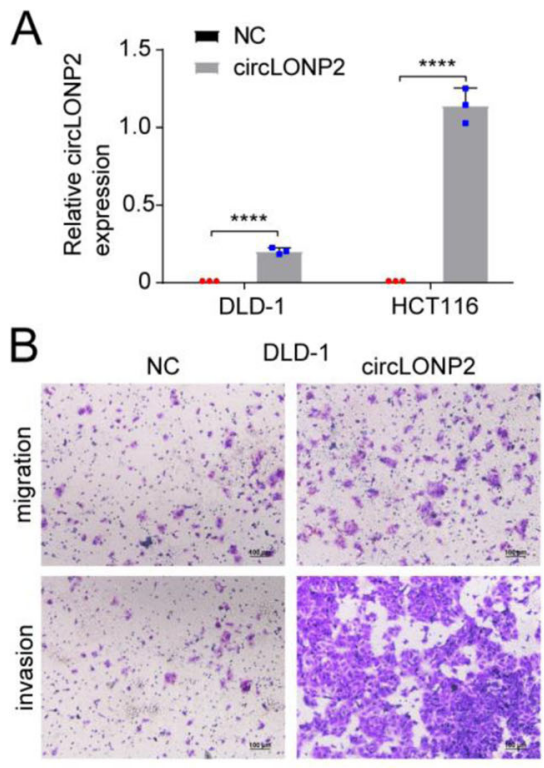

D ASONC DLD-1
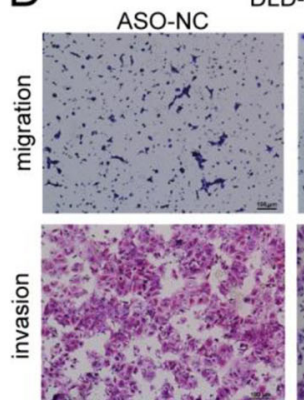

E

NC

circLONP2

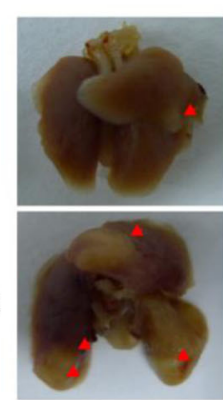

F

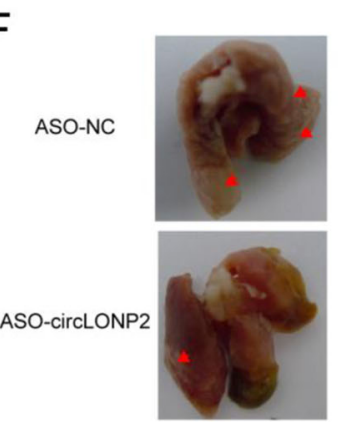

ASO-circLONP2
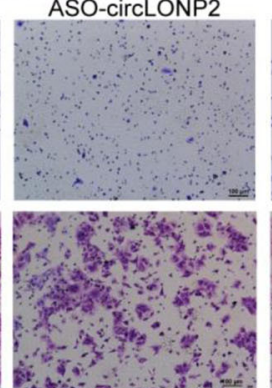

$4 \times$

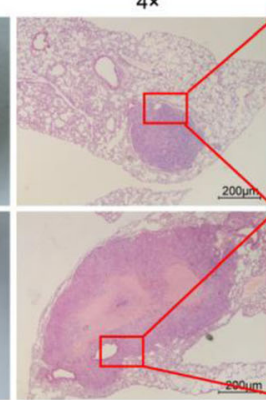

$4 \times$
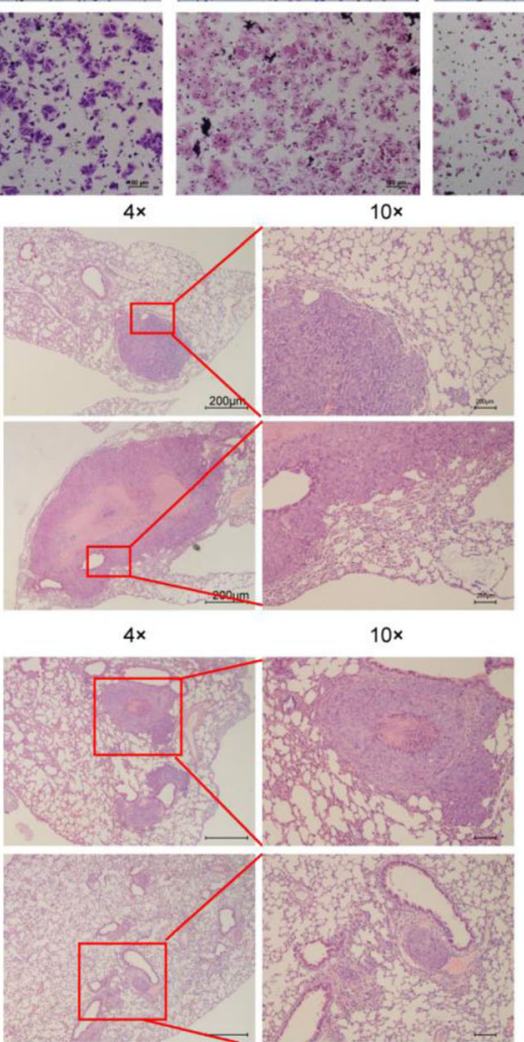

$10 x$
C
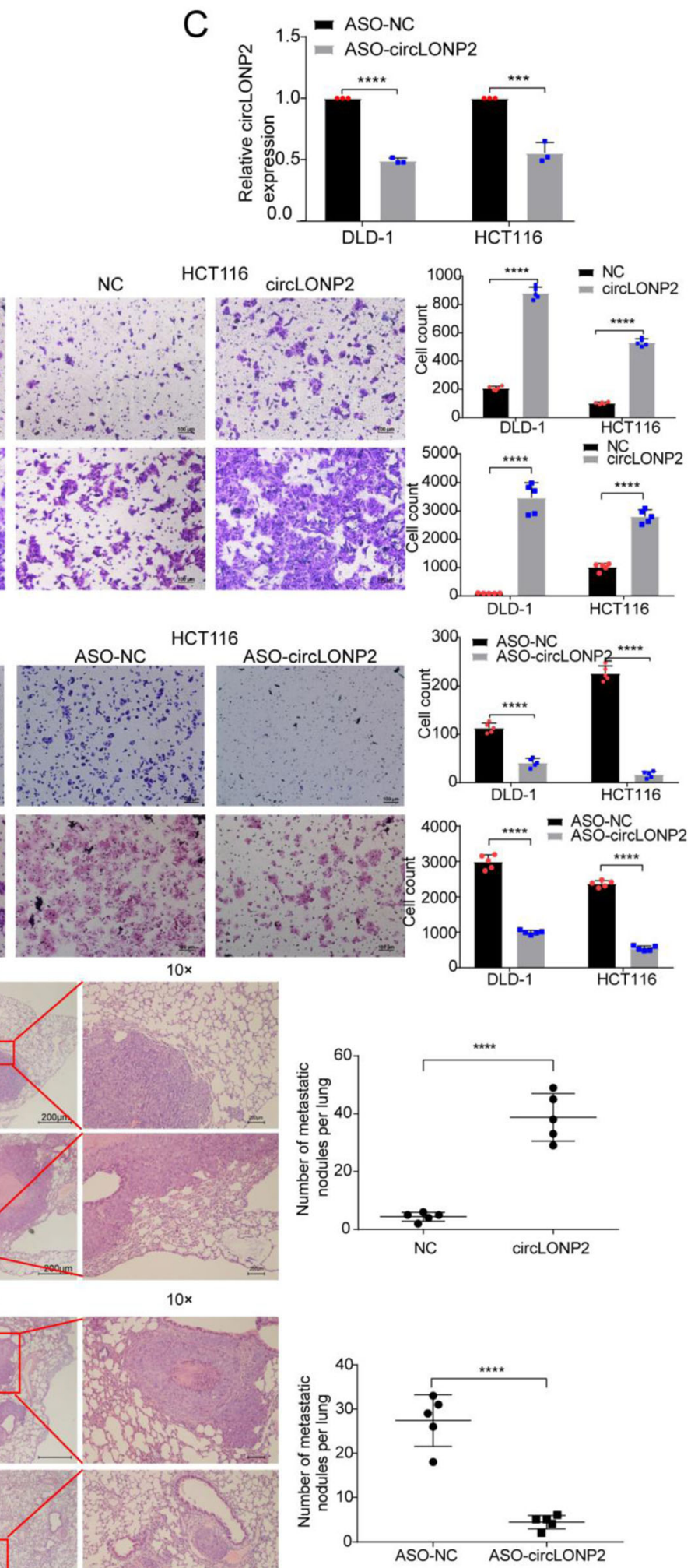

Fig. 4 (See legend on next page.) 
(See figure on previous page.)

Fig. 4 circLONP2 is essential for CRC metastasis. a, b Overexpression of circLONP2 significantly enhanced the migration and invasion ability of CRC cells. c, $\mathbf{d}$ Knockdown of circLONP2 by ASO significantly suppressed the migration and invasion ability of CRC cells. e, $\mathbf{f}$ In vivo tail vein injection model confirmed that overexpression or knockdown of circLONP2 could significantly promote or attenuate CRC cells metastasize to lung, respectively. All detection of circLONP2 by RT-qPCR was normalized to GAPDH. All experiments were repeated for three times, data were shown as mean $\pm \mathrm{SD}$, ${ }^{*} P<0.05$, ${ }^{* *} P<0.01,{ }^{* * *} P<0.001,{ }^{* * *} P<0.0001$ in Mann-Whitney $U$ test $(\mathbf{a}, \mathbf{c}, \mathbf{e}, \mathbf{f})$, or independent Student's $t$ test (b, d) 